Article

\title{
Existence of Solutions for Nonhomogeneous Choquard Equations Involving p-Laplacian
}

\author{
Xiaoyan Shi ${ }^{1}$, Yulin Zhao ${ }^{1, *}$ and Haibo Chen ${ }^{2}$ \\ 1 School of Science, Hunan University of Technology, Zhuzhou 412007, China; xiaoyshi08@126.com \\ 2 School of Mathematics and Statistics, Central South University, Changsha 410083, China; \\ math_chb@csu.edu.cn \\ * Correspondence: zhaoylch@hut.edu.cn
}

Received: 26 August 2019; Accepted: 15 September 2019; Published: 19 September 2019

Abstract: This paper is devoted to investigating a class of nonhomogeneous Choquard equations with perturbation involving p-Laplacian. Under suitable hypotheses about the perturbation term, the existence of at least two nontrivial solutions for the given problems is obtained using Nehari manifold and minimax methods.

Keywords: p-Laplacian; choquard equation; nonhomogeneous; nehari method; minimax methods

\section{Introduction and Main Results}

In this paper we are interested in the following generalized nonlinear Choquard equation with perturbation involving p-Laplacian

$$
-\Delta_{p} u+V(x)|u|^{p-2} u=\left(\int_{\mathfrak{R}^{N}} \frac{|u(y)|^{q}}{|x-y|^{\mu}} d y\right)|u|^{q-2} u+g(x), \quad x \in \mathfrak{R}^{N}
$$

where $N \geq 3,2 \leq p<N, 0<\mu<N, \frac{p}{2}\left(2-\frac{\mu}{N}\right)<q<\frac{p^{*}}{2}\left(2-\frac{\mu}{N}\right), 0<V \in C^{1}\left(\mathfrak{R}^{N}, \mathfrak{R}\right)$, $\Delta_{p}=\operatorname{div}\left(|\nabla u|_{p-2} \nabla u\right)$ is the p-Laplacian operator, and $g: \mathfrak{R}^{N} \rightarrow \mathfrak{R}$ is perturbation. Here $p^{*}=$ $N p /(N-p)$ denotes the Sobolev conjugate of $p$.

The homogeneous, a.e. $g(x) \equiv 0$, which means zero is a solution of problem (1). It was investigated in [1]. A special case of problem (1) is the well-known Choquard-Pekar equation

$$
-\Delta u+u=\left(\frac{1}{|x|^{\mu}} *|u|^{2}\right) u, \quad x \in \mathfrak{R}^{N}
$$

which was investigated by Pekar [2] in relationship with the quantum field theory of a polaron. In particular, when $u$ is a solution to (2), we know that $\phi(x, t)=u(x) e^{-i t}$ is a solitary wave of the following Hartree equation

$$
i \frac{\partial \phi}{\partial t}=-\Delta \phi-\left(\frac{1}{|x|^{\mu}} *|\phi|^{2}\right) \phi, \text { in } \mathfrak{R}^{3} \times \mathfrak{R}_{+}
$$

which was introduced by Choquard in 1976 to describe an electron trapped in its own hole as approximation to Hartree-Fock theory of a one-component plasma; see [3,4]. This equation was also proposed by Penrose in [5] as a model of self-gravitating matter and is usually known in that context as the nonlinear Schrödinger-Newton equation. For more details, discussion about the physical aspects of the problem we refer the readers to [6-11] and the references therein.

From a mathematical point of view, the Choquard-Pekar Equation (2) and its generalizations have been widely studied. Take for instance, Lieb [4] investigated the existence and uniqueness, up 
to translations, of the ground state to problem (2) by using symmetric decreasing rearrangement inequalities. Later, Lions [6] proved the existence of infinitely many radially symmetric solutions to problem (2) via critical point theory. Ackermann [12] established some existence and multiplicity results for a type of periodic Choquard-Pekar equation with nonlocal superlinear part. Further interesting results on Choquard equations may be found in [13-26], the survey [27], and the references therein.

In [15], Ma and Zhao investigated the generalized stationary nonlinear Choquard equation

$$
-\Delta u+u=\left(\int_{\mathfrak{R}^{N}} \frac{|u(y)|^{q}}{|x-y|^{\mu}} d y\right)|u|^{q-2} u, \quad x \in \mathfrak{R}^{N}
$$

where $N \geq 3,0<\mu<N, q \geq 2$ Under the suitable conditions on $\mu, N$, and $q$, which include the classical case, they showed that every positive solution to problem (3) is radially symmetric and monotone decreasing on some point. Using the same condition, Cingolani et al. [9] treated (3) with the case where both the vector and the scalar potential have some symmetries, and they established the regularity and some decay asymptotically at infinity of the ground states to problem (3). In [28], Moroz and Van Schaftingen eliminated this restriction and in the optimal range of parameters they derived the regularity, positivity, and radial symmetry of the ground states, and also gave decay asymptotically at infinity for them.

When the potential $V(x)$ is continuous and bounded below in $\mathfrak{R}^{N}$, Alves and Yang [13] studied the multiplicity and concentration behavior of positive solutions for quasilinear Choquard equation involving p-Laplacian:

$$
-\varepsilon^{p} \Delta_{p} u+V(x)|u|^{p-2} u=\varepsilon^{\mu-N}\left(\int_{\mathfrak{R}^{N}} \frac{Q(y) F(u(y))}{|x-y|^{\mu}} d y\right) Q(x) f(u), x \in \mathfrak{R}^{N}
$$

where $N \geq 3,0<\mu<N, V$, and $Q$ are two continuous real functions in $\mathfrak{R}^{N}, \varepsilon$ is a positive parameter and $F(t)$ be the primate function of $f(t)$, and $\Delta_{p}=\operatorname{div}\left(|\nabla u|_{p-2} \nabla u\right)$ is p-Laplacian operator, $1<p<N$ In [1], suppose that the potential $V$ and the nonlinearity $f$ satisfy suitable assumption, Sun considered the case $\varepsilon=1$ and $Q=1$, and proved the existence of solutions in the level of mountain pass for problem (4). Further, Alves et al. [29] considered a class of generalized Choquard equation with the nonlinearities involving $N$-functions, and they obtained the existence of solutions for the given Choquard equation involving the $\Delta_{\Phi}$-Laplacian operator, where $\Delta_{\Phi}=\operatorname{div}(\phi(|\nabla u|) \nabla u)$ and $\Phi: \mathfrak{R} \rightarrow \mathfrak{R}$ is a $N$-function. Other related results about Choquard equation involving p-Laplacian can be found in [25,30-36] and the references therein.

In 2003, Küpper et al. [37] studied the existence of positive solutions and the bifurcation point for the following Choquard equation

$$
-\Delta u+u=\left(\int_{\mathfrak{R}^{3}} \frac{|u(y)|^{2}}{|x-y|} d y\right) u+\lambda g(x), \quad x \in \mathfrak{R}^{3}
$$

where $g(x) \in H^{-1}\left(\mathfrak{R}^{3}\right), g(x) \geq 0, g(x) \equiv 0$. They proved that there exist positive constants $\lambda_{*}$ and $\lambda_{* *}$ such that problem (5) has at least two positive solutions for $\lambda \in\left(0, \lambda_{*}\right)$, and no positive solution for $\lambda>\lambda_{* *}$ Furthermore, they showed that $\lambda_{*}=\lambda_{* *}$ is a bifurcation point of problem (5).

Very recently, Xie et al. [23] showed the following nonhomogeneous Choquard equation

$$
-\Delta u+V(x) u=\left(\int_{\mathfrak{R}^{N}} \frac{|u(y)|^{q}}{|x-y|^{\mu}} d y\right)|u|^{q-2} u+g(x), \quad x \in \mathfrak{R}^{N}
$$

had two nontrivial solutions if $2-\mu / N<q<(2 N-\mu) /(N-2)$ satisfies the following compactness condition: 
$\left(A_{1}\right) V \in C\left(\mathfrak{R}^{N}, \mathfrak{R}^{+}\right)$is coercive, i.e., $\lim _{|x| \rightarrow+\infty} V(x)=+\infty$.

In [24], Zhang, $\mathrm{Xu}$ and Zhang also investigated the bound and ground states for nonhomogeneous Choquard equation under the following assumption.

$\left(A_{2}\right) V \in C\left(\mathfrak{R}^{N}, \mathfrak{R}^{+}\right), \inf _{\mathfrak{R}^{N}} V>0$, and there exists a positive constant $r$ such that, for any $M>0$, meas $\left\{x \in \mathfrak{R}^{N}:|x-y| \leq r, V(x) \leq M\right\} \rightarrow 0$ as $|y| \rightarrow+\infty$, where meas stands for the Lebesgue measure.

In [38], Shen, Gao and Yang considered a class of critical nonhomogeneous Choquard equation

$$
-\Delta u=\left(\int_{\mathfrak{R}^{N}} \frac{|u(y)|^{q_{*}}}{|x-y|^{\mu}} d y\right)|u|^{q_{*}-2} u+\lambda u+g(x), \quad x \in \Omega
$$

where $\Omega$ is a smooth bounded domain of $\mathfrak{R}^{N}, 0$ in interior of $\Omega, \lambda \in \mathfrak{R}, 0<\mu<N, N \geq 7$, $q_{*}=(2 N-\mu) /(N-2)$ is the upper critical exponent. By applying variational methods, they obtain the existence of multiple solutions for the above problem when $\lambda \in\left(0, \lambda_{1}\right)$, where $\lambda_{1}$ is the first eigenvalue of $-\Delta$. Other related results about non-homogeneous Choquard equation can be found in $[1,29,33,39-43]$ and the references therein.

Our work is motivated by the above work $[23,37,41,44]$ where authors used the structure of associated Nehari manifold to obtain the multiplicity of solutions for the studied problems. Concerning the nonhomogeneous problem, Wang [41] dealt with the problem (1) in the case $p=2, V \equiv 1$ and obtained the multiple solutions of problem (1). In this paper, we investigate the nonhomogeneous problem (1) in case of $2 \leq p<N$ and extend the results in the literatures [23,24,41,44]. The used approach of our paper comes from the literatures $[23,24,41]$. However, owe to dealing with p-Laplacian and nonlocal terms the calculation of our problem will be more complicated.

Before giving our main results, we need the following function spaces. $W^{1, p}\left(\mathfrak{R}^{N}\right)$ is the usual Sobolev space with norm

$$
\|u\|_{1}^{p}=\int_{\mathfrak{R}^{N}}\left(|\nabla u|_{p}+|u|^{p}\right) d x
$$

and $L^{r}\left(\mathfrak{R}^{N}\right)$, for $1 \leq r \leq \infty$ denotes the Lebesgue space with the norm

$$
\|u\|_{r}=\left(\int_{\mathfrak{R}^{N}}|u|^{r} d x\right)^{1 / r}, \text { if } 1 \leq r<\infty
$$

In what follows, we consider the following Banach space

$$
E_{V}=\left\{u \in W^{1, p}\left(\mathfrak{R}^{N}\right): \int_{\mathfrak{R}^{N}} V(x)|u|^{p} d x<+\infty\right\}
$$

endowed with the inner product and norm

$$
\langle u, v\rangle=\int_{\mathfrak{R}^{N}}|\nabla u|^{p-2} \nabla u \nabla v d x+\int_{\mathfrak{R}^{N}}|u|^{p-2} u v d x,\|u\|^{p}=\int_{\mathfrak{R}^{N}}\left(|\nabla u|^{p}+V(x)|u|^{p} d x\right.
$$

Throughout this paper, we assume the following condition on the function $V$.

$\left(A_{0}\right) \quad V \in C\left(\mathfrak{R}^{N}, \mathfrak{R}\right), \inf _{x \in \mathfrak{R}^{N}} V(x)>0$ and there exists a constant $M>0$ such that meas $\left\{x \in \mathfrak{R}^{N}: V(x) \leq M\right\}<\infty$, where meas is the Lebesgue measure.

Now we recall the well-known embedding results in [45] (Lemma 2.1).

Lemma 1. The following statements hold.

(i) There exists a continuous embedding from $W^{1, p}\left(\mathfrak{R}^{N}\right)$ into $L^{r}\left(\mathfrak{R}^{N}\right)$ for any $r \in\left[p, p^{*}\right)$.

(ii) Under the condition $\left(A_{0}\right)$ on $V$, the embedding from $E_{V}$ into $L^{r}\left(\mathfrak{R}^{N}\right)$ is compact for any $r \in\left[p, p^{*}\right)$. 
Denote $S_{r}$ be the best constant of the embedding from $E_{V}$ into $L^{r}\left(\mathfrak{R}^{N}\right)$ as

$$
|u|_{r} \leq S_{r}\|u\|, \quad \forall u \in E_{V}
$$

To obtain our result, we make the following assumption on perturbation term $g$ :

(G). The perturbation function $g \in L^{\frac{2 N q}{2 N(q-1)+\mu}}\left(\mathfrak{R}^{N}\right), g$ is nonzero, and there is a positive constant $\alpha=\alpha\left(N, p, q, \mu, S_{\frac{2 N q}{2 N-\mu}}\right)$, such that $|g| \frac{2 N q}{2 N(q-1)+\mu}<\alpha$.

Obviously, if $g=0$, then we always get a solution for problem (1) that is the trivial solution. Now, the main result of this article reads as follows.

Theorem 1. Suppose $\left(A_{0}\right), g \equiv 0$, and $(\mathrm{G})$ hold. Then problem (1) admits two weak solutions. One of which is a local minimum solution with the ground state energy, and another is bound state solution. In additional, if $g \geq 0$ then the two weak solutions are nonnegative.

This paper is organized as follows. In Section 2, we introduce the variational setting for problem (1) and give some related preliminaries. In Section 3, we study the Palais-Smale sequences and the minimization problems. Finally, we give the proof of Theorem 1 in Section 4.

\section{Variational Setting and Fibering Map Analysis}

This section is devoted to stating the variational setting and giving some lemmas which will be used as tools to prove our main results. The key inequality is the following classical Hardy-Littlewood-Sobolev inequality [3].

Lemma 2. (Hardy-Littlewood-Sobolev inequality [3]). Let $t, s>1$, and $0<\mu<N$ with $\mu / N+1 / s+1 / t=2$ ,$f \in L^{t}\left(R^{N}\right)$ and $g \in L^{s}\left(R^{N}\right)$. Then there exists a constant $C(N, t, \mu, s)$ independent of $f, g$ such that

$$
\int_{\mathfrak{R}^{N}} \int_{\mathfrak{R}^{N}} \frac{f(x) g(y)}{|x-y|^{\mu}} d x d y \leq C(N, t, \mu, s)|f|_{L^{t}} \cdot|g|_{L^{s}}
$$

By the Hardy-Littlewood-Sobolev inequality we have that

$$
\int_{\mathfrak{R}^{N}} \int_{\mathfrak{R}^{N}} \frac{|u(x)| q|u(y)|^{q}}{|x-y|^{\mu}} d x d y
$$

is well defined if $|u|_{q} \in L^{t}\left(R^{N}\right)$ for some $t>1$ satisfying

$$
\frac{\mu}{N}+\frac{2}{t}=2
$$

For we will be working in the space $W^{1, p}\left(\mathfrak{R}^{N}\right)$, by Sobolev embedding theorem we obtain that $q t \in\left[p, p^{*}\right]$, where $p^{*}=N p /(N-p)$; that is

$$
\frac{p}{2}\left(2-\frac{\mu}{N}\right) \leq q \leq \frac{p^{*}}{2}\left(2-\frac{\mu}{N}\right)=\frac{p}{2}\left(\frac{2 N-\mu}{N-p}\right)
$$

Define

$$
q_{l}:=\frac{p}{2}\left(2-\frac{\mu}{N}\right), \text { and } q^{u}:=\frac{p}{2}\left(\frac{2 N-\mu}{N-p}\right)
$$


Therefore, $q_{l}$ and $q^{u}$ are called as lower and upper critical exponents in the sense of the Hardy-Littlewood-Sobolev inequality. We constrain our discussion only when $q \in\left(q_{l}, q^{u}\right)$ We define the energy functional corresponding to problem (1) as

$$
I(u)=\frac{1}{p} \int_{\mathfrak{R}^{N}}\left(|\nabla u|^{p}+V(x)|u|^{p}\right) d x-\frac{1}{2 q} \int_{\mathfrak{R}^{N}} \int_{\mathfrak{R}^{N}} \frac{|u(x)|^{q}|u(y)|^{q}}{|x-y|^{\mu}} d x d y-\int_{\mathfrak{R}^{N}} g(x) u d x, u \in E_{V}
$$

By the condition $(G)$, Hardy-Littlewood-Sobolev inequality and Sobolev inequality, we have

$$
\int_{\mathfrak{R}^{N}} \int_{\mathfrak{R}^{N}} \frac{|u(x)|^{q}|u(y)|^{q}}{|x-y|^{\mu}} d x d y \leq C(N, \mu)\left|u^{q}\right|_{\frac{2 N q}{2 N-\mu}}^{2} \leq C(N, \mu) S_{\frac{2 N q}{2 N-\mu}}^{2 q}\|u\|^{2 q}
$$

and

$$
\int_{\mathfrak{R}^{N}} g(x) u d x \leq|g|_{\frac{2 N q}{2 N(q-1)+\mu}}|u|_{\frac{2 N q}{2 N-\mu}} \leq|g|_{\frac{2 N q}{2 N(q-1)+\mu}} S_{\frac{2 N q}{2 N-\mu}}\|u\|
$$

for any $u^{q} \in L^{r}\left(\mathfrak{R}^{N}\right), r>1, \mu \in(0, N)$ and $q_{l} \leq q \leq q^{u}, g \in L^{\frac{2 N q}{2 N(q-1)+\mu}}\left(\mathfrak{R}^{N}\right)$. Therefore, one knows that $I$ is well defined and $I(u) \in C^{2}\left(E_{V}, \mathfrak{R}\right)$ and its critical points are weak solutions of problem (1). Moreover,

$$
\begin{gathered}
\left\langle I^{\prime}(u), v\right\rangle=\int_{\mathfrak{R}^{N}}\left(|\nabla u|^{p-2} \nabla u \nabla v+V(x)|u|^{p-2} u v\right) d x \\
-\int_{\mathfrak{R}^{N}} \int_{\mathfrak{R}^{N}} \frac{|u(y)|^{q}|u(x)|^{q-2} u(x) v(x)}{|x-y|^{\mu}} d x d y-\int_{\mathfrak{R}^{N}} g(x) v d x
\end{gathered}
$$

for all $v \in E_{V}$. Thus, we will constrain our functional $I$ on the Nehari manifold

$$
\Lambda=\left\{u \in E_{V}:\left\langle I^{\prime}(u), u\right\rangle=0\right\}
$$

Clearly, every nontrivial weak solution of problem (1) belongs to $\Lambda$. Denote $\Psi(u)=\left\langle I^{\prime}(u), u\right\rangle$, so we can see that

$$
\left\langle I^{\prime}(u), u\right\rangle=\|u\|^{p}-\int_{\mathfrak{R}^{N}} \int_{\mathfrak{R}^{N}} \frac{|u(x)|^{q}|u(y)|^{q}}{|x-y|^{\mu}} d x d y-\int_{\mathfrak{R}^{N}} g(x) u(x) d x
$$

and

$$
\left\langle\Psi^{\prime}(u), u\right\rangle=p\|u\|^{p}-2 q \int_{\mathfrak{R}^{N}} \int_{\mathfrak{R}^{N}} \frac{|u(x)|^{q}|u(y)|^{q}}{|x-y|^{\mu}} d x d y-\int_{\mathfrak{R}^{N}} g(x) u(x) d x
$$

Notice that, if $u_{0}$ is a local minimum solution of the functional $I$, one has

$$
\left\langle I^{\prime}\left(u_{0}\right), u_{0}\right\rangle=0, \quad\left\langle\Psi^{\prime}\left(u_{0}\right), u_{0}\right\rangle \geq 0
$$

Thus, we can subdivide the Nehari manifold $\Lambda$ into three parts as follows:

$$
\begin{aligned}
& \Lambda^{+}=\left\{u \in \Lambda:\left\langle\Psi^{\prime}(u), u\right\rangle>0\right\} \\
& \Lambda^{-}=\left\{u \in \Lambda:\left\langle\Psi^{\prime}(u), u\right\rangle<0\right\} \\
& \Lambda^{0}=\left\{u \in \Lambda:\left\langle\Psi^{\prime}(u), u\right\rangle=0\right\}
\end{aligned}
$$

Clearly, only $\Lambda^{0}$ contains the element 0 . It is easy to see that $\Lambda^{0} \cup \Lambda^{+}$and $\Lambda^{0} \cup \Lambda^{-}$are closed subsets of $E_{V}$. In the due course of this paper, we will subsequently give reason to divide the set $\Lambda$ into above three subsets. 
For the convenience of calculations, for $u \in E_{V}$, we denote

$$
\begin{gathered}
A:=A(u)=\int_{\mathfrak{R}^{N}}\left(|\nabla u|^{p-1} \nabla u+V(x)|u|^{p-1} u\right) d x=\|u\|^{p} \\
B:=B(u)=\int_{\mathfrak{R}^{N}} \int_{\mathfrak{R}^{N}} \frac{|u(x)|^{q}|u(y)|^{q}}{|x-y|^{\mu}} d x d y \\
C:=C(u)=\int_{\mathfrak{R}^{N}} g(x) u d x
\end{gathered}
$$

For $u \in E_{V}$, we define the fibering map $\varphi:(0,+\infty) \rightarrow \mathfrak{R}$ as

$$
\varphi(t):=I(t u)=\frac{A}{p} t^{p}-\frac{B}{2 q} t^{2 q}-C t, \quad t>0
$$

From (8) we have

$$
\varphi^{\prime}(t)=\frac{1}{t}\left\langle I^{\prime}(t u), t u\right\rangle=\frac{\Psi(t u)}{t}=A t^{p-1}-B t^{2 q-1}-C
$$

which implies that $u \in \Lambda$ if and only if $\varphi^{\prime}(1)=0$. It is easy to see that $t u \in \Lambda$ with $t>0$ if and only if $\varphi^{\prime}(t)=0$, i.e., $\Lambda=\left\{u \in E_{V}: \varphi^{\prime}(t)=0\right\}$. Moreover,

$$
\varphi^{\prime \prime}(t)=\frac{\left\langle\Psi^{\prime}(t u), t u\right\rangle-\Psi(t u)}{t^{2}}=(p-1) A t^{p-2}-(2 q-1) B t^{2 q-2}
$$

which implies that for $u \in \Lambda,\left\langle\Psi^{\prime}(t u), t u\right\rangle>0$ or $<0$ if and only if $\varphi^{\prime \prime}(t)>0$ or $<0$, respectively. That is to say, from the sign of $\varphi^{\prime \prime}(t)$ the stationary points of $\varphi(t)$ can be divided into three types, namely local minimum, local maximum, and turning point. Thus, $\Lambda^{ \pm}$and $\Lambda^{0}$ can also be written as

$$
\Lambda^{ \pm}=\left\{t u \in \Lambda: \varphi^{\prime \prime}(t)>0 \text { or }<0\right\}, \text { and } \Lambda^{0}=\left\{u \in \Lambda: \varphi^{\prime \prime}(t)=0\right\}
$$

Lemma 3. Assume that $g \equiv 0$ and satisfies $(G)$. Then for any $u \in E_{V} \backslash\{0\}$, there exists a unique $t_{1}=t_{1}(u)>0$ such that $t_{1} u \in \Lambda^{-}$. In particular,

$$
t_{1}>\left[\frac{(p-1) A}{(2 q-1) B}\right]^{1 /(2 q-p)}:=t_{0}
$$

and $I\left(t_{1} u\right)=\max _{t \geq 0} I(t u)$ for $\int_{\mathfrak{R}^{N}} g u d x \leq 0$.

Moreover, if $\int_{\mathfrak{R}^{N}} g u d x>0$, then there exist unique $0<t_{2}=t_{2}(u)<t_{3}=t_{3}(u)$ such that $t_{2} u \in \Lambda^{+}$. In particular, $I\left(t_{3} u\right)=\max _{t \geq t_{2}} I(t u), I\left(t_{2} u\right)=\min _{0 \leq t \leq t_{3}} I(t u)$.

Proof. Set $k(t)=A t^{p-1}-B t^{2 q-1}$, then $\varphi^{\prime}(t)=k(t)-C$ and $k^{\prime}(t)=\varphi^{\prime \prime}(t)$ Obviously, $\lim _{t \rightarrow 0^{+}} k(t)=0$, $\lim _{t \rightarrow+\infty} k(t)=-\infty$ and $k(t)>0$ for $t>0$ sufficiently small. Due to $2 q>p$, if $k^{\prime}\left(t_{0}\right)=0$, then $t_{0}=\left(\frac{(p-1) A}{(2 q-1) B}\right)^{1 /(2 q-p)}$. Thus, we have $k^{\prime}(t)>0$ for $t \in\left(0, t_{0}\right)$, and $k^{\prime}(t)<0$ for $t \in\left(t_{0},+\infty\right)$.

In the case $C=\int_{\mathfrak{R}^{N}} g(x) u d x \leq 0$, there exists a unique $t_{1}$ with $t_{1}>t_{0}$ such that $k\left(t_{1}\right)=\int_{\mathfrak{R}^{N}} g u d x$ and $k^{\prime}\left(t_{1}\right)<0$. Therefore,

$$
\left\langle I^{\prime}\left(t_{1} u\right), t_{1} u\right\rangle=A t_{1}^{p}-B t_{1}{ }^{2 q}-C t_{1}=t_{1}\left(A t_{1}{ }^{p-1}-B t_{1}{ }^{2 q-1}-C\right)=t_{1}\left(k\left(t_{1}\right)-C\right)=0
$$


This implies $t_{1} u \in \Lambda$. Moreover,

$$
\left\langle\Psi^{\prime}\left(t_{1} u\right), t_{1} u\right\rangle=A p t_{1}^{p}-2 B q t_{1}^{2 q}-C t_{1}=(p-1) A t_{1}^{p}-(2 q-1) B t_{1}^{2 q}=t_{1}^{2} k^{\prime}\left(t_{1}\right)<0
$$

which implies that $t_{1} u \in \Lambda^{-}$, and $I\left(t_{1} u\right)=\max _{t \geq 0} I(t u)$.

In the case $C=\int_{\mathfrak{R}^{N}} g(x) u d x>0$, for any $u \in E_{1}$, where $E_{1}=\left\{u \in E_{V}:\|u\|=1\right\}$. By the assumption $(G)$ and $\widetilde{t}_{0}=\widetilde{t}_{0}(u)=\left(\frac{p-1}{(2 q-1) \widetilde{B}(u)}\right)^{1 /(2 q-p)}$, we have

$$
\begin{gathered}
\max _{t \geq 0} \varphi^{\prime}(t) \quad \geq \varphi^{\prime}\left(\widetilde{t_{0}}\right)=\widetilde{t}_{0}^{p-1}-\widetilde{B t}_{0}^{2 q-1}-\widetilde{C} \\
=\left[\frac{p-1}{(2 q-1) \widetilde{B}}\right]^{\frac{p-1}{2 q-p}}-\widetilde{B}\left[\frac{p-1}{(2 q-1) \widetilde{B}}\right]^{\frac{2 q-1}{2 q-p}}-\widetilde{C} \\
=\left[\frac{p-1}{(2 q-1) \widetilde{B}}\right]^{\frac{p-1}{2 q-p}}-\widetilde{B}\left[\frac{p-1}{(2 q-1) \widetilde{B}}\right]^{\frac{2 q-p}{2 q-p}}\left[\frac{p-1}{(2 q-1) \widetilde{B}}\right]^{\frac{p-1}{2 q-p}}-\widetilde{C} \\
=\left[\frac{p-1}{(2 q-1) \widetilde{B}}\right]^{\frac{p-1}{2 q-p}}-\frac{p-1}{2 q-1}\left[\frac{p-1}{(2 q-1) \widetilde{B}}\right]^{\frac{p-1}{2 q-p}}-\widetilde{C} \\
\geq \\
\quad \frac{(p-1)^{\frac{p-1}{2 q-p}}(2 q-p)}{2 q-1}-|g|_{\frac{2 N q}{2 N(q-1)+\mu}} S_{\frac{2 N q}{2 N-\mu}} \\
\quad \geq\left(\alpha-|g|_{\frac{2 N-1}{2 N(q-1)+\mu}}^{2 q-p} S_{\frac{2 N q}{2 N-\mu}}>0\right.
\end{gathered}
$$

where

$$
B_{0}=\sup _{\|u\|=1} \int_{\mathfrak{R}^{N}} \int_{\mathfrak{R}^{N}} \frac{|u(x)|^{q}|u(y)|^{q}}{|x-y|^{\mu}} d x d y, \text { and } \alpha=\alpha\left(N, p, q, \mu, S_{\frac{2 N q}{2 N-\mu}}\right):=\frac{(p-1)^{\frac{p-1}{2 q-p}}(2 q-p)}{(2 q-1)^{\frac{2 q-1}{2 q-p}} B_{0} \frac{p-1}{2 q-p} S_{\frac{2 N q}{2 N-\mu}}}
$$

From (27), we have for $u \in E_{1}$,

$$
\lim _{t \rightarrow 0^{+}} k(t)=0<\int_{\mathfrak{R}^{N}} g(x) u d x \leq|g|_{\frac{2 N q}{2 N(q-1)+\mu}} S_{\frac{2 N q}{2 N-\mu}}\|u\|=|g|_{\frac{2 N q}{2 N(q-1)+\mu}} S_{\frac{2 N q}{2 N-\mu}} \leq k\left(\widetilde{t_{0}}\right)
$$

Hence, there exist unique $0<t_{2}=t_{2}(u)<\tilde{t}_{0}<t_{3}=t_{3}(u)$ such that

$$
k\left(t_{2}\right)=\int_{\mathfrak{R}^{N}} g(x) u d x=k\left(t_{3}\right) \text { and } k^{\prime}\left(t_{3}\right)<0<k^{\prime}\left(t_{2}\right)
$$

Consequently, $t_{2} u \in \Lambda^{+}$and $t_{3} u \in \Lambda^{-}$It is easy to see that $\frac{d}{d t} I(t u)=\varphi^{\prime}(t)=0$ for $t=t_{2}$ or $t=t_{3}$, and $\varphi^{\prime \prime}(t)>0$ for $t \in\left(0, \widetilde{t}_{0}\right)$ and $\varphi^{\prime \prime}(t)<0$ for $\left.t \in \widetilde{t_{0}},+\infty\right)$. Then $I\left(t_{3} u\right)=\max _{t \geq t_{2}} I(t u), I\left(t_{2} u\right)=\min _{0 \leq t \leq t_{3}} I(t u)$. This proof is completed.

Lemma 4. For $g \equiv 0$, the condition $(G)$ is satisfied, then $\Lambda^{0}=\{0\}$.

Proof. To prove $\Lambda^{0}=\{0\}$, we need to show that for any $u \in E_{V} \backslash\{0\}$, the fibering map $\varphi(t)$ has no critical point that is a turning point. For any $u \in \Lambda^{-}$, set $\widetilde{u}=u(\|u\|)^{-1}$, then $\widetilde{u} \in E_{1}$. By the proof of Lemma 3, $k(t)$ has a unique global maximum point $\bar{t}_{0}=\left(\frac{p-1}{(2 q-1) B(\widetilde{u})}\right)^{1 /(2 q-p)}$, and

$$
k\left(\bar{t}_{0}\right)=\frac{(2 q-p)}{2 q-1}\left[\frac{p-1}{(2 q-1) B(\widetilde{u})}\right]^{\frac{p-1}{2 q-p}}:=k_{0}
$$

According to (8)-(10), we deduce that if $0<C(\widetilde{u})<k_{0}$, the equation $\varphi^{\prime}(t)=0$ has exactly two roots $\bar{t}_{1}, \bar{t}_{2}$ satisfying $0<\bar{t}_{1}<\bar{t}_{0}<\bar{t}_{2}$ and if $C(\widetilde{u}) \leq 0, \varphi^{\prime}(t)=0$ has only one point $\bar{t}_{3}$ such that $\bar{t}_{3}>\bar{t}_{0}$. 
Since $\varphi^{\prime \prime}(t)=k^{\prime}(t)$, we have $\varphi^{\prime \prime}\left(\bar{t}_{1}\right)>0, \varphi^{\prime \prime}\left(\bar{t}_{2}\right)<0$ and $\varphi^{\prime \prime}\left(\bar{t}_{3}\right)<0$. Hence, if $0<C(\widetilde{u})<k_{0}$, then $\bar{t}_{1} \widetilde{u} \in \Lambda^{+}, \bar{t}_{2} \widetilde{u} \in \Lambda^{-}$and if $C(\widetilde{u}) \leq 0$, then $\bar{t}_{3} \widetilde{u} \in \Lambda^{-}$. This implies $\Lambda^{ \pm} \cap\left\{u \in E_{V}: \widetilde{u} \in E_{1}, 0<C(\widetilde{u})<k_{0}\right\} \neq$ $\varnothing$ and $\Lambda^{-} \cap\left\{u \in E_{V}: \widetilde{u} \in E_{1}, C(\widetilde{u}) \leq 0\right\} \neq \varnothing$. As a consequence, we infer that $\Lambda^{ \pm}$are nonempty. It is easy to see that for any sign of $C(\widetilde{u})$, critical point of the fibering map $\varphi(t)$ is either a point of local maximum or local minimum which implies $\Lambda^{0}=\{0\}$. Therefore, it remains to show that $k_{0}>C(\widetilde{u})$. By the condition $(G)$ and Lemma 3 we have

$$
k_{0}-C(\widetilde{u})=k\left(\bar{t}_{0}\right)-C(\widetilde{u})=\bar{t}_{0}^{p-1}-B(\widetilde{u})_{0}^{-2 q-1}-C(\widetilde{u})>0
$$

This completes the proof. $\square$

Lemma 5. Assume the condition $(G)$ holds, then $\Lambda^{-}$is closed.

Proof. Let $c l\left(\Lambda^{-}\right)$denote the closure of $\Lambda^{-}$. Due to $c l\left(\Lambda^{-}\right) \subset \Lambda^{-} \cup\{0\}$, it is sufficient to prove that $0 \notin \operatorname{cl}\left(\Lambda^{-}\right)$or equivalently the distance $\operatorname{dist}\left(u, \Lambda^{-}\right)>0$. Set $u \in \Lambda^{-}$and denote $\widetilde{u}=u(\|u\|)^{-1}$, then $\tilde{u} \in E_{1}$. Under the assumption $(G)$ and the proof of Lemma 4 , one has

$C(\widetilde{u})<\widetilde{t}_{0}^{p-1}-B(\widetilde{u}) t_{0}^{2 q-1}=\left[\frac{p-1}{(2 q-1) B(\widetilde{u})}\right]^{\frac{p-1}{2 q-p}}-B(\widetilde{u})\left[\frac{p-1}{(2 q-1) B(\widetilde{u})}\right]^{\frac{2 q-1}{2 q-p}}=\frac{2 q-p}{2 q-1} \cdot\left[\frac{p-1}{(2 q-1) B(\widetilde{u})}\right]^{\frac{p-1}{2 q-p}}=k_{0}$

Moreover, we have that if $C(\widetilde{u}) \leq 0$ then $\varphi^{\prime}(t)=0$ has only one point $\bar{t}_{3}>\bar{t}_{0}$ such that $\bar{t}_{3} \widetilde{u} \in \Lambda^{-}$. Then we have $\bar{t}_{3} \widetilde{u}=u$ with $\|u\|=\bar{t}_{3}>\bar{t}_{0}$. Also, if $0<C(\widetilde{u})<k_{0}$, the equation $\varphi^{\prime}(t)=0$ has exactly two roots $\bar{t}_{1}, \bar{t}_{2}$ with $0<\bar{t}_{1}<\bar{t}_{0}<\bar{t}_{2}$ such that $\bar{t}_{1} \widetilde{u} \in \Lambda^{+}$and $\bar{t}_{2} \widetilde{u} \in \Lambda^{-}$. Hence, we have $\bar{t}_{2} \widetilde{u}=u$ and $\|u\|=\bar{t}_{2}>\bar{t}_{0}$. In a word, for any $u \in \Lambda^{-}$, we get $\|u\|>\bar{t}_{0}$. By (7) we know that $B(\widetilde{u})$ is bounded from above. It follows from definition of $\bar{t}_{0}$ that

$$
\bar{t}_{0}=\left[\frac{p-1}{(2 q-1) B(\widetilde{u})}\right]^{1 /(2 q-p)} \geq\left[\frac{p-1}{(2 q-1) \widetilde{B}_{0}}\right]^{1 /(2 q-p)}:=\tau
$$

where

$$
\widetilde{B}_{0}=\sup _{\|\widetilde{u}\|=1} \int_{\mathfrak{R}^{N}} \int_{\mathfrak{R}^{N}} \frac{|\widetilde{u}(x)|^{q}|\widetilde{u}(y)|^{q}}{|x-y|^{\mu}} d x d y
$$

which implies that $\operatorname{dist}\left(u, \Lambda^{-}\right)=\inf _{u \in \Lambda^{-}}\|u\| \geq \tau>0$. Hence $c l\left(\Lambda^{-}\right)=\Lambda^{-}$and this proves the Lemma.

Lemma 6. Assume $\left(A_{0}\right)$ and $(G)$ hold. Then the functional $I(u)$ is coercive and bounded below on $\Lambda$ Thus $I(u)$ is bounded below on $\Lambda^{+}$and $\Lambda^{-}$.

Proof. Let $u \in \Lambda$, from $\left\langle I^{\prime}(u), u\right\rangle=0$ and (7) we derive that

$$
\begin{aligned}
& I(u)=\frac{1}{p} \int_{\mathfrak{R}^{N}}\left(|\nabla u|^{p}+\right.\left.V(x)|u|^{p}\right) d x-\frac{1}{2 q} \int_{\mathfrak{R}^{N}} \int_{\mathfrak{R}^{N}} \frac{|u(x)|^{q}|u(y)|^{q}}{|x-y|^{\mu}} d x d y-\int_{\mathfrak{R}^{N}} g(x) u d x \\
&=\frac{1}{p} A(u)-\frac{1}{2 q} B(u)-C(u) \\
&=\left(\frac{1}{p}-\frac{1}{2 q}\right) A(u)-\left(1-\frac{1}{2 q}\right) \int_{\mathfrak{R}^{N}} g(x) u d x \\
& \geq \frac{2 q-p}{2 p q}\|u\|^{p}-\frac{2 q-1}{2 q}|g|_{\frac{2 N q}{2 N(q-1)+\mu}} S_{\frac{2 N q}{2 N-\mu}\|u\|}
\end{aligned}
$$

where $S_{\frac{2 N q}{2 N-\mu}}$ denotes the best constant of the embedding from $E_{V}$ into $L^{\frac{2 N q}{2 N-\mu}}\left(\mathfrak{R}^{N}\right)$. It is to see that $I$ is coercive and bounded below in the manifold $\Lambda$. This completes the proof. 


\section{Minimization Problems and Palais-Smale Analysis}

According to Lemma 6, we can define the following two minimization problems:

$$
\begin{aligned}
& i^{-}:=\inf _{u \in \Lambda^{-}} I(u) \\
& i^{+}:=\inf _{u \in \Lambda^{+}} I(u)
\end{aligned}
$$

Clearly, if the infimum of (15) and (16) are achieved, then we can show that they produce a weak solution of our problem (1).

Lemma 7. If $u$ is a local minimizers of $I$ on $\Lambda^{+}$and $\Lambda^{-}$respectively, then $I^{\prime}(u)=0$.

Proof. If $u$ is a local minimizers of $I$ on $\Lambda^{ \pm}$, then $\nabla\left(\left.I\right|_{N^{ \pm}}\right)(u)=0$. Using Theorem 4.1.1 of [46] we infer that there exists Lagrangian multiplier $\lambda \in \mathfrak{R}$ such that

$$
\left\langle I^{\prime}(u), u\right\rangle=\lambda\langle\Psi(u), u\rangle
$$

Since $u \in \Lambda^{ \pm},\left\langle I^{\prime}(u), u\right\rangle=0$ and $\langle\Psi(u), u\rangle \neq 0$. This implies $\lambda=0$. Thus $u$ is a nontrivial weak solution of our problem (1).

By Lemma 6 we know that the problem of investigating solutions of problem (1) can be translated into that of studying minimizers of (15) and (16).

Lemma 8. Assume $\left(A_{0}\right)$ and $(G)$ are satisfied. Then the functional $I(u)$ satisfies $(P S)_{\mathcal{C}}$ condition with $c \in \mathfrak{R}$. That is, if $\left\{u_{n}\right\}$ is a sequence in $E_{V}$ satisfying

$$
I\left(u_{n}\right) \rightarrow c \text { and } I^{\prime}\left(u_{n}\right) \rightarrow 0, \text { as } n \rightarrow+\infty
$$

for some $c \in \mathfrak{R}$, then $\left\{u_{n}\right\}$ possesses a convergent subsequence.

Proof. If $\left\{u_{n}\right\}$ be a sequence in $E_{V}$ satisfies (17), then similar to Lemma 6 we get that $u_{n}$ is bounded in $E_{V}$. Since $E_{V}$ is reflexive Banach space, up to a subsequence, we may assume that $u_{n}$ weakly converges to $u$ in $E_{V}$. By using compact embedding of $E_{V}$ in $L^{r}\left(\mathfrak{R}^{N}\right)$ for $r \in\left[p, p^{*}\right), u_{n}$ strongly converges to $u$ in $L^{r}\left(\mathfrak{R}^{N}\right)$. Since $q \in\left(q_{l}, q^{u}\right)$ and $p<\frac{2 N q}{2 N-\mu}<p^{*}$, it follows from Hardy-Littlewood-Sobolev inequality that

$$
\int_{\mathfrak{R}^{N}} \int_{\mathfrak{R}^{N}} \frac{\left|u_{n}(y)\right|^{q}\left|u_{n}(x)\right|^{q-2} u_{n}(x)\left[u_{n}(x)-u(x)\right]}{|x-y|^{\mu}} d x d y \leq C(N, \mu)\left|u_{n}\right|_{\frac{2 N q}{2 N-\mu}}^{2 q-1}\left|u_{n}-u\right|_{\frac{2 N q}{2 N-\mu}} \rightarrow 0
$$

as $n \rightarrow \infty$. Then, we also get

$$
\int_{\mathfrak{R}^{N}} \int_{\mathfrak{R}^{N}} \frac{\left|u_{n}(y)\right|^{q}\left|u_{n}(x)\right|^{q-2} u_{n}(x)\left[u_{n}(x)-u(x)\right]}{|x-y|^{\mu}} d x d y \rightarrow 0, \quad n \rightarrow \infty
$$

Thus

$$
\begin{gathered}
o(1)=\left\langle I^{\prime}\left(u_{n}\right)-I^{\prime}(u), u_{n}-u\right\rangle \\
=\left\|u_{n}-u\right\|^{p}-\int_{\mathfrak{R}^{N}} \int_{\mathfrak{R}^{N}} \frac{\left|u_{n}(y)\right|^{q}\left|u_{n}(x)\right|^{q-2} u_{n}(x)\left[u_{n}(x)-u(x)\right]}{|x-y|^{\mu}} d x d y \\
+\int_{\mathfrak{R}^{N}} \int_{\mathfrak{R}^{N}} \frac{|u(y)|^{q}|u(x)|^{q-2} u(x)\left[u_{n}(x)-u(x)\right]}{|x-y|^{\mu}} d x d y \\
=\| u_{n}-u||_{p}+o(1)
\end{gathered}
$$

which implies that $u_{n} \rightarrow u$ in $E_{V}$ and consequently ends the proof. 
The following result is an observation regarding the minimizers of $\Lambda^{+}$and $\Lambda^{-}$.

Lemma 9. Assume $\left(A_{0}\right)$ and $(G)$ are satisfied. Then $i^{+}<0$ and $i^{-}>0$.

Proof. Let $u \in \Lambda$, by the proof of Lemma 4 we have that if $0<C(\widetilde{u})<k_{0}$ corresponding to $\widetilde{u}=u(\|u\|)^{-1} \in E_{1}$, then $\varphi^{\prime}(t)=0$ has exactly two roots $\bar{t}_{1}, \bar{t}_{2}$ such that $0<\bar{t}_{1}<\bar{t}_{0}<\bar{t}_{2}$ and $\bar{t}_{1} \widetilde{u} \in \Lambda^{+}$ and $\bar{t}_{2} \bar{u} \in \Lambda^{-}$. Since $\varphi^{\prime}(t)=t^{p-1}-B(\widetilde{u}) t^{2 q-1}-C(\widetilde{u})$, we get that $\lim _{t \rightarrow 0^{+}} \varphi^{\prime}(t)=-C(\widetilde{u})<0$ and $\varphi^{\prime \prime}(t)>0$ for any $t \in\left(0, \bar{t}_{0}\right)$. Due to $\bar{t}_{1}$ is point of local minimum of $\varphi(t)$ and $\bar{t}_{1}>0$, we have that $\varphi\left(\bar{t}_{1}\right)<\lim _{t \rightarrow 0^{+}} \varphi(t)=0$ and then $i^{+} \leq I\left(\bar{t}_{1} \widetilde{u}\right)=\varphi\left(\bar{t}_{1}\right)<0$. Moreover $i:=\inf _{u \in \Lambda} I(u) \leq \inf _{u \in \Lambda^{+}} I(u)=$ $i^{+}<0$.

Now we claim that $i^{-}>0$. In fact, from (7) we know that $B \leq M_{0}\|u\|^{2 q}$, where $M_{0}=C(N, \mu) S_{\frac{2 N q}{2 N-\mu}}^{2 q}$. This implies that there is a positive constant $M_{1}$ which is independent of $u$ such that

$$
\frac{\left(\|u\|_{p}\right)^{2 q /(2 q-p)}}{B^{p /(2 q-p)}}=\frac{A^{2 q /(2 q-p)}}{B^{p /(2 q-p)}} \geq M_{1}
$$

By the given assumption and (18) we discuss $\varphi\left(t_{0}\right)$ corresponding to $u$ as

$$
\begin{gathered}
\varphi\left(t_{0}\right)=\frac{1}{p}\|u\| p t_{0}^{p}-\frac{1}{2 q} B t_{0}^{2 q}-C t_{0} \\
=\frac{A}{p}\left[\frac{(p-1) A}{(2 q-1) B}\right]^{p /(2 q-p)}-\frac{B}{2 q}\left[\frac{(p-1) A}{(2 q-1) B}\right]^{2 q /(2 q-p)}-C\left[\frac{(p-1) A}{(2 q-1) B}\right]^{1 /(2 q-p)} \\
=\frac{(2 q-p)(2 q+p-1)}{2 p q(2 q-1)} \cdot \frac{A^{2 q /(2 q-p)}}{B^{p /(2 q-p)}}-C\left(\frac{p-1}{2 q-1}\right)^{1 /(2 q-p)} \cdot \frac{A^{1 /(2 q-p)}}{B^{1 /(2 q-p)}} \\
\geq \frac{(2 q-p)(2 q+p-1)}{2 p q(2 q-1)} \cdot \frac{A^{2 q /(2 q-p)}}{B^{p /(2 q-p)}} \\
\geq \frac{(2 q-p)(2 q+p-1)}{2 p q(2 q-1)} \cdot M_{1}:=M_{*}
\end{gathered}
$$

where the positive constant $M_{*}$ is independent of $u$. Hence,

$$
i^{-}=\inf _{u \in \Lambda \backslash\{0\}} \max \{I(u)\} \geq \inf _{u \in \Lambda \backslash\{0\}} \varphi\left(t_{0}\right) \geq M_{*}>0 .
$$

This completes the proof.

Now we study the nature of minimizing sequences for the functional $I(u)$. Using the idea of [44] to obtain a $(P S)_{i^{+}}$sequence from the minimization sequence of our problem (1). The following lemma is a consequence of Lemma 4.

Lemma 10. Assume $\left(A_{0}\right)$ and $(G)$ hold. Then for $u \in \Lambda^{+}$, there exists a constant $\rho>0$ and a differentiable function $\eta^{+}: B(0, \rho) \rightarrow \mathfrak{R}_{+}:=(0,+\infty)$ such that $\eta^{+}(0)=1, \eta^{+}(w)(u-w) \in \Lambda^{+}$, and

$$
\begin{gathered}
\left\langle\left(\eta^{+}\right)^{\prime}(0), w\right\rangle=M^{*}\left[p \int_{\mathfrak{R}^{N}}\left(|\nabla u|^{p-2} \nabla u \nabla w+V(x)|u|^{p-2} u w\right) d x\right. \\
\left.\quad-2 q \int_{\mathfrak{R}^{N}} \int_{\mathfrak{R}^{N}} \frac{|u(y)|^{||^{\mid}|u(x)|^{q-2} u(x) w(x)}}{|x-y|^{\mu}} d x d y-\int_{\mathfrak{R}^{N}} g w d x\right]
\end{gathered}
$$

for any $w \in B(0, \rho)$, where $B(0, \rho)$ denotes the ball centered at 0 with radius $\rho$, and $M^{*}=$ $\left[(p-1)\|u\|^{p}-(2 q-1) B(u)\right]^{-1}$.

Proof. Fixing a function $u \in \Lambda^{+}$, we define a $C^{1}$ mapping $\Phi: \mathfrak{R} \times E_{V} \rightarrow \mathfrak{R}$ as follows

$$
\Phi(t, w)=t^{p-1}\|u-w\|^{p}-t^{2 q-1} \int_{\mathfrak{R}^{N}} \int_{\mathfrak{R}^{N}} \frac{|(u-w)(y)|^{q}|(u-w)(x)|^{q}}{|x-y|^{\mu}} d x d y-\int_{\mathfrak{R}^{N}} g(x)(u-w) d x
$$


Notice that $\Phi(1,0)=\left\langle I^{\prime}(u), u\right\rangle=0$. Moreover

$$
\Phi(t, 0)=A t^{p-1}-t^{2 q-1} B-C=\varphi^{\prime}(t)
$$

where $\varphi$ is the fibering map defined in (8). Since $u \in \Lambda^{+}$, we have $\varphi^{\prime \prime}(1)>0$, and then so $\Phi_{t}(1,0)=\varphi^{\prime \prime}(1) \neq 0$. By Applying the implicit function theorem at point $(1,0)$, we get that there is $\rho=\rho(u)>0$ and a differentiable function $\eta^{+}: B(0, \rho) \rightarrow \mathfrak{R}_{+}$such that $\eta^{+}(0)=1, \eta^{+}(w)(u-w) \in \Lambda$ for any $w \in B(0, \rho)$, and

$$
\left\langle\left(\eta^{+}\right)^{\prime}(0), w\right\rangle=-\frac{\left\langle\Phi_{w}(1,0), w\right\rangle}{\Phi_{t}(1,0)}
$$

Now we only show that $\eta^{+}(w)(u-w) \in \Lambda^{+}$for any $w \in B(0, \rho)$. In fact, from Lemma 5 it follows that $\Lambda^{-} \cup \Lambda^{0}$ is closed, then the distance $\operatorname{dist}\left(u, \Lambda^{-} \cup \Lambda^{0}\right)>0$. Since the function $\eta^{+}(w)(u-w)$ is continuous with respect to $w$, taking $\rho=\rho(u)>0$ sufficiently small, such that

$$
\left\|\eta^{+}(w)(u-w)-u\right\|<\frac{1}{4} \operatorname{dist}\left(u, \Lambda^{-} \cup \Lambda^{0}\right), \forall w \in B(0, \rho)
$$

Then $\eta^{+}(w)(u-w)$ does not belong to $\Lambda^{-} \cup \Lambda^{0}$. Thus $\eta^{+}(w)(u-w) \in \Lambda^{+}$. Finally, (19) can be obtained by direct differentiating $\Phi\left(w, \eta^{+}(w)\right)=0$ with respect to $w$.

This completes the proof.

To derive a sequence $(P S)_{i^{-}}$from the minimizing sequence of our problem (1), similar to Lemma 10 we can obtain the following proposition.

Proposition 1. If $\left(A_{0}\right)$ and $(G)$ are satisfied. Then for $u \in \Lambda^{-}$, there exists a constant $\rho>0$ and a differentiable function $\eta^{-}: B(0, \rho) \rightarrow \mathfrak{R}_{+}$such that $\eta^{-}(0)=1, \eta^{-}(w)(u-w) \in \Lambda^{-}$, and

$$
\begin{gathered}
\left\langle\left(\eta^{-}\right)^{\prime}(0), w\right\rangle=M^{*}\left[p \int_{\mathfrak{R}^{N}}\left(|\nabla u|^{p-2} \nabla u \nabla w+V(x)|u|^{p-2} u w\right) d x\right. \\
\left.-2 q \int_{\mathfrak{R}^{N}} \int_{\mathfrak{R}^{N}} \frac{|u(y)|^{q}|u(x)|^{q-2} u(x) w(x)}{|x-y|^{\mu}} d x d y-\int_{\mathfrak{R}^{N}} g w d x\right]
\end{gathered}
$$

for any $w \in B(0, \rho)$, and $M^{*}=\left[(p-1)\|u\|^{p}-(2 q-1) B(u)\right]^{-1}$.

Lemma 11. If $\left(A_{0}\right)$ and $(G)$ are satisfied. There exists a positive constant $M$ such that

$$
-\frac{(2 q-p)(p-1)}{2 p q} \theta^{\frac{p}{p-1}} \leq i=\inf _{u \in \Lambda} I(u) \leq-\frac{(2 p q-2 q-p)(2 q-p)}{4 p q^{2}} \cdot M
$$

where $\theta=\frac{2 q-1}{2 q-p}|g|_{\frac{2 N q}{2 N(q-1)}} S_{\frac{2 N q}{2 N-\mu}}$

Proof. For any $u \in \Lambda$, According to (13) one has

$$
I(u) \geq \frac{2 q-p}{2 p q}\|u\|^{p}-\frac{2 q-1}{2 q}|g|_{\frac{2 N q}{2 N(q-1)+\mu}} S_{\frac{2 N q}{2 N-\mu}}\|u\| \geq-\frac{(2 q-p)(p-1)}{2 p q} \theta^{\frac{p}{p-1}}
$$

Thus,

$$
i \geq-\frac{(2 q-p)(p-1)}{2 p q} \theta^{\frac{p}{p-1}}
$$

On the other hand, set $u_{0} \in \Lambda$ be the unique solution of the following equation

$$
-\Delta_{p} u+V(x)|u|_{p-1} u=g(x), \quad \forall x \in \mathfrak{R}^{N}
$$


Due to $g \neq 0, \int_{\mathfrak{R}^{N}} g(x) u_{0} d x=\left\|u_{0}\right\|^{p}>0$. Then by Lemma 4 , there exists $t_{1}>0$ such that $t_{1} u_{0} \in \Lambda^{+}$. Therefore,

$$
\begin{gathered}
I\left(t_{1} u_{0}\right)=\frac{1-p}{p}\left\|u_{0}\right\|^{p} t_{1}^{p}+\frac{2 q-1}{2 q} t_{1}^{2 q} B\left(u_{0}\right) \\
\quad<\frac{1-p}{p}\left\|u_{0}\right\|^{p} t_{1}^{p}+\frac{p(2 q-1)}{4 q^{2}} t_{1}^{p}\left\|u_{0}\right\|^{p} \\
\quad=-\frac{(2 p q-2 q-p)(2 q-p)}{4 p q^{2}} t_{1}^{p}\left\|u_{0}\right\|^{p}<0
\end{gathered}
$$

Choose $M=t_{1}^{p}\left\|u_{0}\right\|^{p}$ we obtain the result.

Lemma 12. If $\left(A_{0}\right)$ and $(G)$ are satisfied, then there exists a sequence $\left\{u_{n}\right\} \subset \Lambda^{+}$such that $I\left(u_{n}\right) \rightarrow i^{+}$and $I^{\prime}\left(u_{n}\right) \rightarrow 0$ as $n \rightarrow \infty$.

Proof. From Lemma 6, we already show that $I$ is bounded from below on $\Lambda$, and $\Lambda^{+} \cup\{0\}$ is closed in $\Lambda$. Obviously Ekeland's variational principle (see [44]) applies to the minimization problem (16). It admits a minimizing sequence $\left\{u_{n}\right\} \subset \Lambda^{+}$such that

(i) $I\left(u_{n}\right)<\inf _{u \in \Lambda^{+} \cup\{0\}}\{I(u)\}+\frac{1}{n}$, and

(ii) $I(w) \geq I\left(u_{n}\right)-\frac{1}{n}\left\|w-u_{n}\right\|, \forall w \in \Lambda^{+} \cup\{0\}$

Then by (i) we have

$$
I(u)=\frac{2 q-p}{p}\left\|u_{n}\right\|^{p}-\frac{2 q-1}{2 q} \int_{\mathfrak{R}^{N}} g(x) u_{n} d x<i+\frac{1}{n}
$$

for $n$ large enough. This together with Lemma 11 shows

$$
\int_{\mathfrak{R}^{N}} g(x) u_{n} d x \geq \frac{(2 p q-2 q-p)(2 q-p)}{2 p q(2 q-1)} M>0
$$

which implies $u_{n} \neq 0$ for any $n$. By Lemma 4, we know $i \leq \inf _{u \in \Lambda^{+}} I(u)=i^{+}<0$. Notice that $I(0)=0$, then $\inf _{u \in \Lambda^{+} \cup\{0\}}\{I(u)\}=i^{+}$. Hence $I\left(u_{n}\right) \rightarrow i^{+}$as $n \rightarrow \infty$, and we can assume that $u_{n} \in \Lambda^{+}$. Then $\left\|u_{n}\right\|^{p}=B\left(u_{n}\right)+C\left(u_{n}\right)$. Furthermore, we deduce from (13) and (i) that

$$
i^{+}+\frac{1}{n} \geq I\left(u_{n}\right) \geq \frac{2 q-p}{2 p q}\|u\|^{p}-\frac{2 q-1}{2 q}|g|_{\frac{2 N q}{2 N(q-1)+\mu}} S_{\frac{2 N q}{2 N-\mu}\|u\|}
$$

which implies that $\left\{u_{n}\right\}$ is bounded. Now we claim that $\inf _{n}\left\|u_{n}\right\| \geq \xi>0$ for some constant $\xi$. In fact, if not, by (23), $I\left(u_{n}\right) \rightarrow 0$, as $n \rightarrow \infty$. Using (23) which is a contradiction to first assertion. Therefore, there exist positive constants $\xi_{2}>\xi_{1}$ such that

$$
\xi_{1} \leq\left\|u_{n}\right\| \leq \xi_{2}
$$

Now to finish the proof, we only need to show that $I^{\prime}\left(u_{n}\right) \rightarrow 0$, as $n \rightarrow \infty$. By Lemma 10, for each $n$, we get a differentiable function $\eta_{n}^{+}: B(0, \varepsilon) \rightarrow \mathfrak{R}_{+}$for $\varepsilon>0$ as follows

$$
\eta_{n}^{+}(\delta):=\eta_{n}^{+}\left(\delta h_{n}\right),-\varepsilon<\delta<\varepsilon
$$

where $h_{n}=\frac{I^{\prime}\left(u_{n}\right)}{\left\|I^{\prime}\left(u_{n}\right)\right\|}$. According to Lemma 10, we get $\eta_{n}^{+}(0)=1$, and

$$
w_{\delta}:=\eta_{n}^{+}(\delta)\left[u_{n}-\delta h_{n}\right] \in \Lambda^{+}
$$

By Taylor's expansion and (ii), since $w_{\delta} \in \Lambda^{+}$we have

$$
\begin{aligned}
& \frac{1}{n}\left\|w_{\delta}-u_{n}\right\| \geq I\left(u_{n}\right)-I\left(w_{\delta}\right) \\
& =\left(1-\eta_{n}^{+}(\delta)\right)\left\langle I^{\prime}\left(w_{\delta}\right), u_{n}\right\rangle+\delta \eta_{n}^{+}(\delta)\left\langle I^{\prime}\left(w_{\delta}\right), h_{n}\right\rangle+o\left(\left\|w_{\delta}-u_{n}\right\|\right)
\end{aligned}
$$


which implies

$$
\left(\frac{1}{n}+o(1)\right)\left\|w_{\delta}-u_{n}\right\| \geq\left(1-\eta_{n}^{+}(\delta)\right)\left\langle I^{\prime}\left(w_{\delta}\right), u_{n}\right\rangle+\delta \eta_{n}^{+}(\delta)\left\langle I^{\prime}\left(w_{\delta}\right), h_{n}\right\rangle
$$

Dividing (25) by $\delta$ for $\delta \neq 0$ and passing to the limit as $\delta \rightarrow 0$, we obtain

$$
\left(\frac{1}{n}+o(1)\right)\left(1+\left|\left(\eta_{n}^{+}\right)^{\prime}(0)\right|\left\|u_{n}\right\|\right) \geq-\left(\eta_{n}^{+}\right)^{\prime}(0)\left\langle I^{\prime}\left(u_{n}\right), u_{n}\right\rangle+\left\|I^{\prime}\left(u_{n}\right)\right\|
$$

Since $u_{n} \in \Lambda^{+}$, it follows from (26) that

$$
\left\|I^{\prime}\left(u_{n}\right)\right\| \leq\left(\frac{1}{n}+o(1)\right)\left(1+\left|\left(\eta_{n}^{+}\right)^{\prime}(0)\right| \cdot\left\|u_{n}\right\|\right)
$$

From (24) we know that $u_{n}$ is bounded. Then it remains to prove that $\left|\left(\eta_{n}^{+}\right)^{\prime}(0)\right|$ is uniformly bounded with respect to $n$. In fact, according to the definition of $\eta_{n}^{+}$and Lemma 5 , we have

$$
\begin{aligned}
\left\langle\left(\eta_{n}^{+}\right)^{\prime}(0), h_{n}\right\rangle= & \frac{1}{(p-1)|| u_{n} \|_{p^{-}-(2 q-1) B\left(u_{n}\right)}}\left[p \int_{\mathfrak{R}^{N}}\left(\left|\nabla u_{n}\right|^{p-2} \nabla u_{n} \nabla h_{n}+V(x)\left|u_{n}\right|^{p-2} u_{n} h_{n}\right) d x\right. \\
& \left.-2 q \int_{\mathfrak{R}^{N}} \int_{\mathfrak{R}^{N}} \frac{\left|u_{n}(y)\right|^{q}\left|u_{n}(x)\right|^{q-2} u_{n}(x) h_{n}(x)}{|x-y|^{\mu}} d x d y-\int_{\mathfrak{R}^{N}} g h_{n} d x\right]
\end{aligned}
$$

By the boundedness of $u_{n}$ and (28), we say that there exists a constant $\lambda$ such that

$$
\left|\left(\eta_{n}^{+}\right)^{\prime}(0)\right|=\left|\left\langle\left(\eta_{n}^{+}\right)^{\prime}(0), h_{n}\right\rangle\right| \leq \frac{\lambda}{(p-1)\left\|u_{n}\right\|_{p}-(2 q-1) B\left(u_{n}\right)}
$$

Therefore, it remains to show that $\chi\left(u_{n}\right):=(p-1)\left\|u_{n}\right\|_{p}-(2 q-1) B\left(u_{n}\right)$ possesses a positive lower bound.

To prove the existence of positive lower bound of $\chi\left(u_{n}\right)$, passing to a subsequence, we assume

$$
\chi\left(u_{n}\right)=(p-1)\left\|u_{n}\right\|^{p}-(2 q-1) B\left(u_{n}\right)=o(1), n \rightarrow \infty
$$

Since $u_{n} \in \Lambda^{+}$, we obtain

$$
\left\|u_{n}\right\|^{p}-B\left(u_{n}\right)=C\left(u_{n}\right)
$$

This along with (29) gives

$$
C\left(u_{n}\right)=\frac{2 q-p}{2 q-1}\left\|u_{n}\right\|^{p}+o(1)
$$

It follows from the condition $(G)$ that there is a sufficiently small $\mu>0$ such that $|g|_{\frac{2 N q}{2 N(q-1)+\mu}} \leq$ $(1-\mu) \alpha$. Similarly to the proof of (12), we have

$$
C(u)<\frac{2 q-p}{2 q-1}(1-\mu)\left(\frac{p-1}{(2 q-1) B(u)}\right)^{\frac{p-1}{2 q-p}}
$$

for any $u \in E_{1}$. Therefore, by the principle of homogeneity,

$$
\frac{2 q-p}{2 q-1}+\frac{o(1)}{\left\|u_{n}\right\|^{p}}=\frac{C\left(u_{n}\right)}{\left\|u_{n}\right\|^{p}}<\frac{2 q-p}{2 q-1}(1-\tau)\left(\frac{(p-1)\left\|u_{n}\right\|^{p}}{(2 q-1) B\left(u_{n}\right)}\right)^{\frac{p-1}{2 q-p}}
$$

If $\left\|u_{n}\right\| \rightarrow 0$, then similar to (7) one has $C\left(u_{n}\right) \rightarrow 0$. Therefore

$$
i^{+}+o_{n}(1)=I\left(u_{\mathrm{n}}\right)-\frac{1}{2 q}\left\langle I^{\prime}\left(u_{\mathrm{n}}\right), u_{n}\right\rangle=\frac{2 q-p}{2 p q}\left\|u_{n}\right\|^{p}-\frac{2 q-1}{2 q} C\left(u_{n}\right) \rightarrow 0
$$


which is a contradiction with $i^{+}<0$. Thus $\left\|u_{n}\right\| \rightarrow 0$, as $n \rightarrow \infty$. Consequently, from (30)-(32) we can deduce that

$$
\frac{2 q-p}{2 q-1} \leq \frac{2 q-p}{2 q-1}(1-\mu), n \rightarrow \infty
$$

which is a contradiction. Therefore, we conclude that $I^{\prime}\left(u_{n}\right) \rightarrow 0$, as $n \rightarrow \infty$. The proof is completed.

Proposition 2. Under assumptions $\left(A_{0}\right)$ and $(G)$, there exists a sequence $\left\{\hat{u}_{n}\right\} \subset \Lambda^{-}$such that $I\left(\hat{u}_{n}\right) \rightarrow i^{-}$ and $I^{\prime}\left(\hat{u}_{n}\right) \rightarrow 0$ as $n \rightarrow \infty$.

Proof. By Lemma 5 we know that $\Lambda^{-}$is closed. Thus, by Ekeland's variational principle on $\Lambda^{-}$we get a sequence $\left\{\hat{u}_{n}\right\} \subset \Lambda^{-}$such that

(iii) $I\left(\hat{u}_{n}\right)<\inf _{u \in \Lambda^{-}}\{I(u)\}+\frac{1}{n}$, and (iv) $I(w) \geq I\left(\hat{u}_{n}\right)-\frac{1}{n}\left\|w-\hat{u}_{n}\right\|, \forall w \in \Lambda^{-}$.

From (24) we know that $\hat{u}_{n}$ is bounded. By coercivity of $I,\left\{\hat{u}_{n}\right\}$ forms a bounded sequence in $\Lambda$. Moreover, from Lemma 5 we know that $\inf _{u \in \Lambda^{-}}\|u\| \geq \tau>0$, which implies that $\Lambda^{-}$stays away from the origin. Then using Proposition 1 and following the proof of Lemma 12 we conclude the result.

\section{The Proof of Theorem 1}

In this section, we show that the minimums are achieved for $i^{+}$and $i^{-}$, and also give the proof of Theorem 1 .

Proposition 3. Assume $g \neq 0,\left(A_{0}\right)$ and $(G)$ are satisfied. Then $i$ can be achieved at point $u_{*} \in \Lambda$, which is a weak solution of problem (1). Moreover, $u_{*} \in \Lambda^{+}$and $u_{*}$ is a local minimum for I on $E_{V}$.

Proof. By Lemma 8, there exists a sequence $\left\{u_{n}\right\} \subset \Lambda$ such that $I\left(u_{n}\right) \rightarrow i$ and $I^{\prime}\left(u_{n}\right) \rightarrow 0$ as $n \rightarrow \infty$. Set $u_{*}$ be the weak limit of the sequence $\left\{u_{n}\right\}$ on $E_{V}$, then $u_{n} \in \Lambda$ satisfies (22) we get

$$
\int_{\mathfrak{R}^{N}} g(x) u_{*}(x) d x>0
$$

On the other hand, $I^{\prime}\left(u_{n}\right) \rightarrow 0$ as $n \rightarrow \infty$ implies that

$$
\left\langle I^{\prime}\left(u_{*}\right), v\right\rangle=0 \text {, for every } v \in \Lambda
$$

i.e., $u_{*}$ is a weak solution of problem (1). In particular, $u_{*} \in \Lambda$, and

$$
i \leq I\left(u_{*}\right) \leq \lim _{n \rightarrow+\infty} \inf \left\{I\left(u_{n}\right)\right\}=i
$$

This implies that $u_{*}$ is the minimum of $I$ over $E_{V}$.

For $u_{*} \in \Lambda$ be such that $i=I\left(u_{*}\right)$, using Lemma 9 we have $I\left(u_{*}\right)<0$. Then we get $u_{*} \neq 0$. Therefore $u_{*}$ is a nontrivial weak solution of problem (1). Since (33) holds, applying Lemma 4 we see that there exist $t_{1}, t_{2}>0$ such that $u_{1}:=t_{1} u_{*} \in \Lambda^{+}$and $t_{2} u_{*} \in \Lambda^{-}$. We claim that $t_{1}=1$ i.e., $u_{*} \in \Lambda^{+}$. If $t_{1}<1$, then $t_{2}=1$ which means $u_{*} \in \Lambda^{-}$. Now $I\left(t_{1} u_{*}\right) \leq I\left(u_{*}\right)=i<0$ which is a contradiction with $t_{1} u_{*} \in \Lambda^{+}$.

Next we will prove that $u_{*}$ is also a local minimum of $I$ on $E_{V}$. Obviously, for any $u \in \Lambda$ with $C(u)>0$ we can deduce that

$$
I\left(\widetilde{t_{2}} u\right) \leq I(\widetilde{t} u) \text { for any } \widetilde{t} \in\left(0, t_{0}\right)
$$

where $t_{0}=\left(\frac{(p-1) A}{(2 q-1) B}\right)^{1 /(2 q-p)}, \widetilde{t}_{2}$ is corresponding to $u$. Moreover, if $u=u_{*}$ then

$$
\widetilde{t}_{2}=1<\hat{t}_{0}=\left[\frac{(p-1) A\left(u_{*}\right)}{(2 q-1) B\left(u_{*}\right)}\right]^{1 /(2 q-p)}
$$


Taking $\rho>0$ small enough so that

$$
1<\hat{t}_{w}=\left[\frac{(p-1) A\left(u_{*}-w\right)}{(2 q-1) B\left(u_{*}-w\right)}\right]^{1 /(2 q-p)}\|w\|<\rho
$$

Thus, it follows from Lemma 10 that there exists a differentiable map $\eta^{+}: B(0, \rho) \rightarrow \mathfrak{R}_{+}$such that $\eta^{+}(w)\left(u_{*}-w\right) \in \Lambda^{+}$for $\|w\|<\rho$ small. Then for any $\tilde{t} \in\left(0, \hat{t}_{w}\right)$ we have

$$
I\left(\widetilde{t}\left(u_{*}-w\right)\right) \geq I\left(\eta^{+}(w)\left(u_{*}-w\right)\right) \geq I\left(u_{*}\right)
$$

Since (34) holds, taking $\widetilde{t}=1$ in (35) we get $I\left(u_{*}\right) \leq I\left(u_{*}-w\right)$ for $\|w\|<\rho$, which implies that $u_{*}$ is a local minimum of $I$ on $E_{V}$. The proof is completed.

Proof of Theorem 1. Firstly, we deal with the minimization problem (16). According to Proposition 3 , we only need to show that there exist a nonnegative solution on $\Lambda^{+}$if $g \geq 0$. Assume $g \geq 0$, from the proof of Lemma 3, it is easy to see that $B\left(u_{*}\right)=B\left(\left|u_{*}\right|\right)$ and $C\left(u_{*}\right) \leq C\left(\left|u_{*}\right|\right)$. Moreover, it follows from the proof of Lemma 4 that there exists $t_{1}>0$ such that $t_{1}\left|u_{*}\right| \in \Lambda^{+}$and $t_{1}\left|u_{*}\right|>0$. If $\varphi_{u}(t)$ denotes the fibering map corresponding to $u \in E_{V}$ as introduced in Section 2, we have $\varphi^{\prime}{ }_{\left|u_{*}\right|}(1) \leq \varphi^{\prime}{ }_{u_{*}}(1)=0$. Since $t_{1}$ is the point of local minimum of $\varphi_{\left|u_{*}\right|}(t)$ for $t \in\left(0, t_{0}\left(\left|u_{*}\right|\right)\right)$, where

$$
t_{0}\left(\left|u_{*}\right|\right)=\left[\frac{(p-1) A\left(\left|u_{*}\right|\right)}{(2 q-1) B\left(\left|u_{*}\right|\right)}\right]^{1 /(2 q-p)}
$$

and $t_{1} \geq 1$. Consequently, we have that $I\left(t_{1}\left|u_{*}\right|\right) \leq I\left(\left|u_{*}\right|\right)$. Then

$$
i^{+} \leq I\left(t_{1}\left|u_{*}\right|\right) \leq I\left(\left|u_{*}\right|\right) \leq I\left(u_{*}\right)=i^{+}
$$

This means that $t_{1}\left|u_{*}\right|$ solves the minimization problem (16). Therefore, we find a nonnegative solution for problem (1) using the maximum principle.

Now we show that the infimum $i^{-}$is achieved and the minimizer is second weak solution of problem (1). Consider the minimization problem (15). From Proposition 2, we know that there exists a sequence $\left\{\hat{u}_{n}\right\} \subset \Lambda^{-}$such that $I\left(\hat{u}_{n}\right) \rightarrow i^{-}$and $I^{\prime}\left(\hat{u}_{n}\right) \rightarrow 0$ as $n \rightarrow \infty$. By Lemma 4 , we get that there exists $\widetilde{u}_{*} \in \operatorname{cl}\left(\Lambda^{-}\right)=\Lambda^{-}$such that $I\left(\widetilde{u}_{*}\right)=i^{-}, I^{\prime}\left(\widetilde{u}_{*}\right)=0$. Therefore, Lemma 7 implies that $\widetilde{u}_{*}$ is a weak solution of problem (1). In addition, if $g \geq 0$, it follows from the proof of Lemma 4 and Proposition 1 that there exists $t_{2}>0$ such that $t_{2}\left|\widetilde{u}_{*}\right| \in \Lambda^{-}$. Let

$$
t_{0}\left(\left|\widetilde{u}_{*}\right|\right)=\left[\frac{(p-1) A\left(\left|\widetilde{u}_{*}\right|\right)}{(2 q-1) B\left(\left|\widetilde{u}_{*}\right|\right)}\right]^{1 /(2 q-p)}
$$

then since $\widetilde{u}_{*} \in \Lambda^{-}$, taking account of the graph of the fibering map corresponding to $\widetilde{u}_{*}$ we can deduce that

$$
i^{-} \leq I\left(t_{2}\left|\widetilde{u}_{*}\right|\right) \leq I\left(t_{2} \widetilde{u}_{*}\right) \leq \max _{\left.t \geq t_{0}\left(\widetilde{u}_{*}\right)\right)}\left\{I\left(t_{2} \widetilde{u}_{*}\right)\right\}=I\left(\widetilde{u}_{*}\right)=i^{-}
$$

This means that $t_{2}\left|\widetilde{u}_{*}\right|$ solves the minimization problem (15) and then we know that it is a nonnegative weak solution of problem (1) using the maximum principle again. Due to $\Lambda^{+} \cap \Lambda^{-}=\varnothing$ and Lemma 9 shows that $i^{+}<i^{-}$, then $u_{*} \neq \widetilde{u}_{*}$. This ends the proof.

\section{Conclusions}

In this work, we study a class of nonhomogeneous Choquard equations with perturbation involving $\mathrm{p}$-Laplacian. We give sufficient conditions of the existence of at least two nontrivial solutions 
for problems (1). Next it is worth investigating infinitely many solutions for nonhomogeneous Choquard equations involving p-Laplacian.

Author Contributions: Supervision, Y.Z. and H.C.; Writing-original draft, X.S.; Writing-review \& editing, X.S. and Y.Z.

Funding: The research was supported by Hunan Provincial Natural Science Foundation of China (No.2019JJ40068).

Acknowledgments: The authors thank the anonymous referees for their careful reading and helpful suggestions, which help to improve the quality of this paper.

Conflicts of Interest: The authors declare no conflict of interest.

\section{References}

1. Sun, X. The existence of solutions for Choquard type equation. Acta Math Sci. 2018, 38, 54-60.

2. Pekar, S. Untersuchung Uber Die Elektronentheorie der Kristalle; Akademie Verlag: Berlin, Germany, 1954.

3. Lieb, E.H.; Loss, M. Analysis. Graduate Studies in Mathematics; American Mathematical Society: Providence, RI, USA, 2001.

4. Lieb, E.H. Existence and uniqueness of the minimizing solution of Choquard's nonlinear equation. Stud. Appl. Math. 1977, 57, 93-105. [CrossRef]

5. Penrose, R. Quantum computation, entanglement and state reduction. Philos. Trans. Roy. Soc. 1998, 356, 1927-1939. [CrossRef]

6. Lions, P.L. The Choquard equation and related questions. Nonlinear Anal. 1980, 4, 1063-1072. [CrossRef]

7. Choquard, P.; Stubbe, J.; Vuray, M. Stationary solutions of the Schröinger-Newton model-an ODE approach. Differ. Integral Equ. 2008, 21, 665-679.

8. Moroz, I.M.; Penrose, R.; Tod, P. Spherically-symmetric solutions of the Schröinger-Newton Equations. Class. Quantum Gravity 1998, 15, 2733-2742. [CrossRef]

9. Cingolani, S.; Clapp, M.; Secchi, S. Multiple solutions to a magnetic nonlinear Choquard equation. Z. Angew. Math. Phys. 2012, 63, 233-248. [CrossRef]

10. Cingolani, S.; Clapp, M.; Secchi, S. Intertwining semiclassical solutions to a Schrödinger-New ton system. Discret. Contin. Dyn. Syst. 2013, 6, 891-908.

11. Cingolani, S.; Secchi, S. Ground states for the pseudo relativistic Hartree equation with external potential. Proc. R. Soc. Edinb. Sect. A 2015, 145, 73-90. [CrossRef]

12. Ackermann, N. On a periodic Schrödinger equation with nonlocal superlinear part. Math. Z. 2004, 248, 423-443. [CrossRef]

13. Alves, C.O.; Yang, M.B. Existence of semiclassical ground state solutions for a generalized Choquard equation. J. Differ. Equ. 2014, 257, 4133-4164. [CrossRef]

14. Moroz, V.; Van Schaftingen, J. Nonexistence and optimal decay of super solutions to Choquard equations in exterior domains. J. Differ. Equ. 2013, 254, 3089-3145. [CrossRef]

15. Ma, L.; Zhao, L. Classication of positive solitary solutions of the nonlinear Choquard equation. Arch. Ration. Mech. Anal. 2010, 195, 455-467. [CrossRef]

16. Moroz, V.; Van Schaftingen, J. Existence of ground states for a class of nonlinear Choquard Equations. T. Am. Math. Soc. 2015, 367, 6557-6579. [CrossRef]

17. Gao, F.S.; Yang, M.B. The Brezis-Nirenberg type critical problem for nonlinear Choquard Equation. Sci. China Math. 2018, 61, 1219-1242. [CrossRef]

18. Clapp, M.; Salazar, D. Positive and sign changing solutions to a nonlinear Choquard equation. J. Math. Anal. Appl. 2013, 407, 1-15. [CrossRef]

19. Alves, C.O.; Yang, M.B. Investigating the multiplicity and concentration behavior of solutions for quasilinear Choquard equation via penalization method. Proc. R. Soc. Edinb. Sect. A 2016, 146, 23-58. [CrossRef]

20. Alves, C.O.; Cassani, D.; Tarsi, C.; Yang, M. Existence and concentration of ground state solutions for a critical nonlocal Schrödinger equation in $R^{2}$. J. Differ. Equ. 2016, 261, 1933-1972. [CrossRef]

21. Alves, C.O.; Gao, F.; Squassina, M.; Yang, M. Singularly perturbed critical Choquard equations. J. Differ. Equ. 2017, 263, 3943-3988. [CrossRef]

22. Su, Y.; Chen, H.B. The minimizing problem involving p-Laplacian and Hardy-Littlewood-Sobolev upper critical exponent. arXiv 2018, arXiv:1805.10986. [CrossRef] 
23. Xie, T.; Xiao, L.; Wang, J. Existence of multiple positive solutions for Choquard equation with perturbation. Adv. Math. Phys. 2015, 2015, 760157. [CrossRef]

24. Zhang, H.; Xu, J.X.; Zhang, F.B. Bound and Ground states for a concave-convex generaliezed Choquard equation. Acta Appl. Math. 2017, 147, 81-93. [CrossRef]

25. Wang, T. Ground state solutions for Choquard type equations with a singular potential. Electron. J. Differ. Equ. 2017, 52, 1-14.

26. Li, F.Y.; Long, L.; Huang, Y.Y.; Zhang, Z.P. Ground state for Choquard equation with doubly critical growth nonlinearity. Electron. J. Qual. Theory Differ. Equ. 2019, 33, 1-15. [CrossRef]

27. Moroz, V.; Van Schaftingen, J. A guide to the Choquard equation. J. Fixed Point Theory Appl. 2017, 19, 773-813. [CrossRef]

28. Moroz, V.; Van Schaftingen, J. Ground states of nonlinear Choquard equations: Existence, qualitative properties and decay asymptotics. J. Funct. Anal. 2013, 265, 153-184. [CrossRef]

29. Alves, C.O.; Râdulescu, V.D.; Tavares, L.S. Generalized Choquard Equations Driven by Nonhomogeneous Operators. Mediterr. J. Math. 2019, 16, 20. [CrossRef]

30. Azzollini, A.; d'Avenia, P.; Pomponio, A. Quasilinear elliptic equations in $\mathrm{R}^{\mathrm{N}}$ via variational methods and Orlicz-Sobolev embeddings. Calc. Var. Partial Differ. Equ. 2014, 49, 197-213. [CrossRef]

31. Alves, C.O.; Da Silva, A.R. Multiplicity and concentration behavior of solutions for a quasilinear problem involving N-functions via penalization method. Electron. J. Differ. Equ. 2016, 158, 1-24.

32. Alves, C.O.; Da Silva, A.R. Multiplicity and concentration of positive solutions for a class of quasilinear problems through Orlicz-Sobolev space. J. Math. Phys. 2016, 57, 11502. [CrossRef]

33. Tuhina, M.; Konijeti, S. On doubly nonlocal p-fractional coupled elliptic system, Topol. Meth. Nonlinear Anal. 2018, 51, 609-636.

34. Abdellaoui, B.; Attar, A.; Bentifour, R. On the fractional p-Laplacian equations with weight and general datum. Adv. Nonlinear Anal. 2019, 8, 144-174. [CrossRef]

35. Pucci, P.; Xiang, M.Q.; Zhang, B.L. Existence results for Schrödinger-Choquard-Kirchhoff equations involving the fractional p-Laplacian. Adv. Nonlinear Anal. 2019, 12, 253-275. [CrossRef]

36. Ambrosio, V. On the multiplicity and concentration of positive solutions for a p-fractional Choquard equation in $\mathrm{R}^{\mathrm{N}}$. Comput. Math. Appl. 2019. [CrossRef]

37. Küpper, T.; Zhang, Z.; Xia, H. Multiple positive solutions and bifurcation for an equation related to Choquard's equation. Proc. Edinb. Math. Soc. 2003, 46, 597-607. [CrossRef]

38. Shen, Z.F.; Gao, F.S.; Yang, M.B. Multiple solutions for nonhomogeneous Choquard eqution involving Hardy-Littlewood-Sobolev critical exponent. Z. Angew. Math. Phys. 2017, 68, 61. [CrossRef]

39. Cerami, G.; Vaira, G. Multiple solutions for nonhomogeneous Schrödinger-Maxwell and Klein-GordonMaxwell equations on $\mathrm{R}^{3}$. J. Differ. Equ. 2010, 248, 521-543. [CrossRef]

40. Chen, S.J.; Tang, C.L. Multiple solutions for nonhomogeneous Schrödinger-Maxwell and KleinGordon-Maxwell equations on $\mathrm{R}^{3}$. Nonlinear Differ. Equ. Appl. 2010, 17, 559-574. [CrossRef]

41. Wang, L. Multiple solutions for nonhomogeneous Choquard equations. Electron. J. Differ. Equ. 2018, 172, $1-27$.

42. Su, Y.; Chen, H.B. Existence of nontrivial solutions for a perturbation of Choquard equation with Hardy-Littlewood-Sobolev upper critical exponent. Electron. J. Differ. Equ. 2018, 2018, 1-25.

43. Zhang, Z.J. Multiple solutions of nonhomogeneous Chouquard's equations. Acta Math. Appl. Sin. 2001, 17, $47-52$.

44. Tarantello, G. On nonhomogeneous elliptic equations involving critical Sobolev exponent. In Annales de l'Institut Henri Poincare (C) Non Linear Analysis; Elsevier Masson: Paris, France, 1992.

45. Lin, X.; Tang, X.H. Existence of infinitely many solutions for $\mathrm{p}$-Laplacian equations in $\mathrm{R}^{\mathrm{N}}$. Nonlinear Anal. 2013, 92, 72-81. [CrossRef]

46. Chang, K.C. Methods in Nonlinear Analysis; Springer: Berlin/Heidelberg, Germany, 2005.

(C) 2019 by the authors. Licensee MDPI, Basel, Switzerland. This article is an open access article distributed under the terms and conditions of the Creative Commons Attribution (CC BY) license (http://creativecommons.org/licenses/by/4.0/). 